\title{
Interactive comment on "Improving regional air quality predictions in the Indo-Gangetic Plain-Case study of an intensive pollution episode in November 2017' by Behrooz Roozitalab et al.
}

\section{Behrooz Roozitalab et al.}

behrooz-roozitalab@uiowa.edu

Received and published: 16 November 2020

The comment was uploaded in the form of a supplement:

https://acp.copernicus.org/preprints/acp-2020-744/acp-2020-744-AC3-supplement.pdf

Interactive comment on Atmos. Chem. Phys. Discuss., https://doi.org/10.5194/acp-2020-744, 2020. 